\title{
Fixed Point Theorems for Ćirić-Berinde Type Contractive Multivalued Mappings
}

\author{
Seong-Hoon Cho \\ Department of Mathematics, Hanseo University, Chungnam 356-706, Republic of Korea \\ Correspondence should be addressed to Seong-Hoon Cho; shcho@hanseo.ac.kr
}

Received 24 April 2014; Revised 24 September 2014; Accepted 28 October 2014

Academic Editor: Salvador Romaguera

Copyright ( 2015 Seong-Hoon Cho. This is an open access article distributed under the Creative Commons Attribution License, which permits unrestricted use, distribution, and reproduction in any medium, provided the original work is properly cited.

We give a Ćirić-Berinde type contractive condition for multivalued mappings and analyze the existence of fixed point for these mappings.

\section{Introduction and Preliminaries}

In 2012, Samet et al. [1] introduced the notions of $\alpha-\psi$ contractive mapping and $\alpha$-admissible mappings in metric spaces and obtained corresponding fixed point results, which are generalizations of ordered fixed point results (see [1]). Since then, by using their idea, some authors investigated fixed point results in the field. Asl et al. [2] extended some of results in [1] to multivalued mappings by introducing the notions of $\alpha_{*}-\psi$-contractive mapping and $\alpha_{*}$-admissible mapping.

Recently, Salimi et al. [3] modified the notions of $\alpha-\psi$ contractive mapping and $\alpha$-admissible mappings by introducing another function $\eta$. And then, they gave generalizations of the results of Samet et al. [1] and Karapinar and Samet [4]. Hussain et al. [5] extended these modified notions to multivalued mappings. That is, they introduced the notion of $\alpha-\eta$-contractive multifunctions and gave fixed point results for these multifunctions.

Very recently, Ali et al. [6] generalized and extended the notion of $\alpha-\psi$-contractive mapping by introducing the notion of $(\alpha, \psi, \xi)$-contractive multivalued mappings and obtained fixed point theorems for these mappings in complete metric spaces.

The purpose of this paper is to introduce the notion of Cirić-Berinde type contractive multivalued mappings and to generalize and extend the notion of $\alpha-\eta$-contractive multifunctions and to establish fixed point theorems for ĆirićBerinde type contractive multivalued mappings.
Let $(X, d)$ be a metric space. We denote by $C B(X)$ the class of nonempty closed and bounded subsets of $X$ and by $C L(X)$ the class of nonempty closed subsets of $X$. Let $H(\cdot, \cdot)$ be the generalized Hausdorff distance on $C L(X)$; that is, for all $A, B \in C L(X)$,

$$
H(A, B)=\left\{\begin{array}{c}
\max \left\{\begin{array}{c}
\left.\sup _{a \in A}(a, B), \sup _{b \in B}(b, A)\right\}, \\
\text { if the maximum exists, }
\end{array}\right. \\
\infty, \quad \text { otherwise, }
\end{array}\right.
$$

where $d(a, B)=\inf \{d(a, b): b \in B\}$ is the distance from point $a$ to subset $B$.

For $A, B \in C L(X)$, let $D(A, B)=\sup _{x \in A} \inf _{y \in B} d(x, y)$.

Then, we have $D(A, B) \leq H(A, B)$ for all $A, B \in C L(X)$.

From now on, we denote by

$$
\begin{gathered}
M(x, y)=\max \{d(x, y), d(x, T x), d(y, T y), \\
\left.\frac{1}{2}\{d(x, T y)+d(y, T x)\}\right\}
\end{gathered}
$$

for a multivalued map $T: X \rightarrow C L(X)$ and $x, y \in X$.

We denote by $\Xi$ the class of all functions $\xi:[0, \infty) \rightarrow$ $[0, \infty)$ such that

(1) $\xi$ is continuous;

(2) $\xi$ is nondecreasing on $[0, \infty)$;

(3) $\xi(t)=0$ if and only if $t=0$; 
(4) $\xi$ is subadditive.

Also, we denote by $\Psi$ the family of all nondecreasing functions $\psi:[0, \infty) \rightarrow[0, \infty)$ such that $\sum_{n=1}^{\infty} \psi^{n}(t)<\infty$ for each $t>0$, where $\psi^{n}$ is the $n$th iterate of $\psi$.

Note that if $\psi \in \Psi$, then $\psi(0)=0$ and $0<\psi(t)<t$ for all $t>0$.

Let $(X, d)$ be a metric space, and let $\alpha: X \times X \rightarrow[0, \infty)$ be a function.

We consider the following conditions:

(1) for any sequence $\left\{x_{n}\right\}$ in $X$ with $\alpha\left(x_{n}, x_{n+1}\right) \geq 1$ for all $n \in \mathbb{N}$ and $\lim _{n \rightarrow \infty} x_{n}=x$, we have

$$
\alpha\left(x_{n}, x\right) \geq 1 \quad \forall n \in \mathbb{N}
$$

(2) for any sequence $\left\{x_{n}\right\}$ in $X$ with $\alpha\left(x_{n}, x_{n+1}\right) \geq 1$ for all $n \in \mathbb{N}$ and a cluster point $x$ of $\left\{x_{n}\right\}$, we have

$$
\lim _{n \rightarrow \infty} \inf \alpha\left(x_{n}, x\right) \geq 1
$$

(3) for any sequence $\left\{x_{n}\right\}$ in $X$ with $\alpha\left(x_{n}, x_{n+1}\right) \geq 1$ for all $n \in \mathbb{N}$ and a cluster point $x$ of $\left\{x_{n}\right\}$, there exists a subsequence $\left\{x_{n(k)}\right\}$ of $\left\{x_{n}\right\}$ such that

$$
\alpha\left(x_{n(k)}, x\right) \geq 1 \quad \forall k \in \mathbb{N}
$$

Remark 1. (1) implies (2) and (2) implies (3).

Note that if $(X, d)$ is a metric space and $\xi \in \Xi$, then $(X, \xi$ 。 $d)$ is a metric space.

Let $(X, d)$ be a metric space, and let $T: X \rightarrow C L(X)$ be a multivalued mapping. Then, we say that

(1) $T$ is called $\alpha_{*}$-admissible [2] if

$$
\alpha(x, y) \geq 1 \quad \text { implies } \alpha_{*}(T x, T y) \geq 1 \text {, }
$$

where $\alpha_{*}(T x, T y)=\inf \{\alpha(a, b): a \in T x, b \in T y\} ;$

(2) $T$ is called $\alpha$-admissible [7] if, for each $x \in X$ and $y \in$ Tx with $\alpha(x, y) \geq 1$, we have $\alpha(y, z) \geq 1$ for all $z \in$ $T y$.

Lemma 2. Let $(X, d)$ be a metric space, and let $T: X \rightarrow$ $C L(X)$ be a multivalued mapping. If $T$ is $\alpha_{*}$-admissible, then it is $\alpha$-admissible.

Proof. Suppose that $T$ is an $\alpha_{*}$-admissible mapping.

Let $x \in X$ and $y \in T x$ be such that $\alpha(x, y) \geq 1$.

Let $z \in T y$ be given.

Since $T$ is $\alpha_{*}$-admissible, $\alpha(y, z) \geq \alpha_{*}(T x, T y) \geq 1$.

Lemma 3. Let $(X, d)$ be a metric space, and let $\xi \in \Xi$ and $B \in C L(X)$.

If $a \in X$ and $\xi(d(a, B))<c$, then there exists $b \in B$ such that $\xi(d(a, b))<c$.
Proof. Let $\epsilon=c-\xi(d(a, B))$.

Since $\xi(d(a, B))<c$ and $\xi \circ d$ is metric on $X$, there exists $b \in B$ such that $\xi(d(a, b))<\xi(d(a, B))+\epsilon$ by definition of infimum. Hence, $\xi(d(a, b))<c$.

Let $(X, d)$ be a metric space.

A function $f: X \rightarrow[0, \infty)$ is called upper semicontinuous if, for each $x \in X$ and $\left\{x_{n}\right\} \subset X$ with $\lim _{n \rightarrow \infty} x_{n}=x$, we have $\lim _{n \rightarrow \infty} f\left(x_{n}\right) \leq f(x)$.

A function $f: X \rightarrow[0, \infty)$ is called lower semicontinuous if, for each $x \in X$ and $\left\{x_{n}\right\} \subset X$ with $\lim _{n \rightarrow \infty} x_{n}=x$, we have $f(x) \leq \lim _{n \rightarrow \infty} f\left(x_{n}\right)$.

For a multivalued map $T: X \rightarrow C L(X)$, let $f_{T}: X \rightarrow$ $[0, \infty)$ be a function defined by $f_{T}(x)=d(x, T x)$.

\section{Fixed Point Theorems}

In this section, we establish fixed point theorems for ĆirićBerinde type contractive multivalued mappings.

Theorem 4. Let $(X, d)$ be a complete metric space, and let $\alpha$ : $X \times X \rightarrow[0, \infty)$ be a function. Suppose that a multivalued mapping $T: X \rightarrow C L(X)$ is $\alpha$-admissible.

Assume that, for all $x, y \in X, \alpha(x, y) \geq 1$ implies

$\xi(H(T x, T y)) \leq \psi(\xi(M(x, y)))+L \xi(d(y, T x))$,

where $L \geq 0, \xi \in \Xi$, and $\psi \in \Psi$ is strictly increasing.

Also, suppose that the following are satisfied:

(1) there exists $x_{0} \in X$ and $x_{1} \in T x_{0}$ such that $\alpha\left(x_{0}, x_{1}\right) \geq$ 1 ;

(2) either $T$ is continuous or $f_{T}$ is lower semicontinuous.

Then $T$ has a fixed point in $X$.

Proof. Let $x_{0} \in X$ and $x_{1} \in T x_{0}$ be such that $\alpha\left(x_{0}, x_{1}\right) \geq 1$.

Let $c$ be a real number with $\xi\left(d\left(x_{0}, x_{1}\right)\right)<\xi(c)$.

If $x_{0}=x_{1}$, then $x_{1}$ is a fixed point.

Let $x_{0} \neq x_{1}$.

If $x_{1} \in T x_{1}$, then $x_{1}$ is a fixed point. Let $x_{1} \notin T x_{1}$. Then $d\left(x_{1}, T x_{1}\right)>0$.

From (7) we obtain

$$
\begin{aligned}
& 0<\xi\left(d\left(x_{1}, T x_{1}\right)\right) \\
& \leq \xi\left(H\left(T x_{0}, T x_{1}\right)\right) \\
& \leq \psi\left(\xi \left(\operatorname { m a x } \left\{d\left(x_{0}, x_{1}\right), d\left(x_{0}, T x_{0}\right), d\left(x_{1}, T x_{1}\right),\right.\right.\right. \\
& \left.\left.\left.\frac{1}{2}\left\{d\left(x_{0}, T x_{1}\right)+d\left(x_{1}, T x_{0}\right)\right\}\right\}\right)\right) \\
& +L \xi\left(d\left(x_{1}, T x_{0}\right)\right) \\
& \leq \psi\left(\xi \left(\operatorname { m a x } \left\{d\left(x_{0}, x_{1}\right), d\left(x_{0}, x_{1}\right), d\left(x_{1}, T x_{1}\right),\right.\right.\right. \\
& \left.\left.\left.\frac{1}{2}\left\{d\left(x_{0}, T x_{1}\right)+d\left(x_{1}, x_{1}\right)\right\}\right\}\right)\right) \\
& +L \xi\left(d\left(x_{1}, x_{1}\right)\right)
\end{aligned}
$$




$$
\begin{gathered}
\leq \psi\left(\xi \left(\operatorname { m a x } \left\{d\left(x_{0}, x_{1}\right), d\left(x_{0}, x_{1}\right), d\left(x_{1}, T x_{1}\right),\right.\right.\right. \\
\left.\left.\left.\frac{1}{2}\left\{d\left(x_{0}, x_{1}\right)+d\left(x_{1}, T x_{1}\right)\right\}\right\}\right)\right) \\
=\psi\left(\xi\left(\max \left\{d\left(x_{0}, x_{1}\right), d\left(x_{1}, T x_{1}\right)\right\}\right)\right) .
\end{gathered}
$$

If $\max \left\{d\left(x_{0}, x_{1}\right), d\left(x_{1}, T x_{1}\right)\right\}=d\left(x_{1}, T x_{1}\right)$, then we have $0<\xi\left(d\left(x_{1}, T x_{1}\right)\right) \leq \psi\left(\xi\left(d\left(x_{1}, T x_{1}\right)\right)\right)<\xi\left(d\left(x_{1}, T x_{1}\right)\right)$, which is a contradiction.

Thus, $\max \left\{d\left(x_{0}, x_{1}\right), d\left(x_{1}, T x_{1}\right)\right\}=d\left(x_{0}, x_{1}\right)$, and hence we have

$$
0<\xi\left(d\left(x_{1}, T x_{1}\right)\right) \leq \psi\left(\xi\left(d\left(x_{0}, x_{1}\right)\right)\right)<\psi(\xi(c)) .
$$

Hence, there exists $x_{2} \in T x_{1}$ such that

$$
\xi\left(d\left(x_{1}, x_{2}\right)\right)<\psi(\xi(c)) .
$$

Since $T$ is $\alpha$-admissible, from condition (1) and $x_{2} \in T x_{1}$, we have

$$
\alpha\left(x_{1}, x_{2}\right) \geq 1 .
$$

If $x_{2} \in T x_{2}$, then $x_{2}$ is a fixed point. Let $x_{2} \notin T x_{2}$.

Then $d\left(x_{2}, T x_{2}\right)>0$, and so $\xi\left(d\left(x_{2}, T x_{2}\right)\right)>0$.

From (7) we obtain

$$
\begin{aligned}
& 0<\xi\left(d\left(x_{2}, T x_{2}\right)\right) \\
& \leq \xi\left(H\left(T x_{1}, T x_{2}\right)\right) \\
& \leq \psi\left(\xi \left(\operatorname { m a x } \left\{d\left(x_{1}, x_{2}\right), d\left(x_{1}, T x_{1}\right), d\left(x_{2}, T x_{2}\right),\right.\right.\right. \\
& \left.\left.\left.\frac{1}{2}\left\{d\left(x_{1}, T x_{2}\right)+d\left(x_{2}, T x_{1}\right)\right\}\right\}\right)\right) \\
& +L \xi\left(d\left(x_{2}, T x_{1}\right)\right) \\
& \leq \psi\left(\xi \left(\operatorname { m a x } \left\{d\left(x_{1}, x_{2}\right), d\left(x_{1}, x_{2}\right), d\left(x_{2}, T x_{2}\right),\right.\right.\right. \\
& \left.\left.\left.\frac{1}{2}\left\{d\left(x_{1}, T x_{2}\right)+d\left(x_{2}, x_{2}\right)\right\}\right\}\right)\right) \\
& +L \xi\left(d\left(x_{2}, x_{2}\right)\right) \\
& \leq \psi\left(\xi \left(\operatorname { m a x } \left\{d\left(x_{1}, x_{2}\right), d\left(x_{1}, x_{2}\right), d\left(x_{2}, T x_{2}\right),\right.\right.\right. \\
& \left.\left.\left.\frac{1}{2}\left\{d\left(x_{1}, x_{2}\right)+d\left(x_{2}, T x_{2}\right)\right\}\right\}\right)\right) \\
& =\psi\left(\xi\left(\max \left\{d\left(x_{1}, x_{2}\right), d\left(x_{2}, T x_{2}\right)\right\}\right)\right) .
\end{aligned}
$$

If $\max \left\{d\left(x_{1}, x_{2}\right), d\left(x_{2}, T x_{2}\right)\right\}=d\left(x_{2}, T x_{2}\right)$, then we have $\xi\left(d\left(x_{2}, T x_{2}\right)\right) \leq \psi\left(\xi\left(d\left(x_{2}, T x_{2}\right)\right)\right)<\xi\left(d\left(x_{2}, T x_{2}\right)\right)$, which is a contradiction.

Thus, $\max \left\{d\left(x_{1}, x_{2}\right), d\left(x_{2}, T x_{2}\right)\right\}=d\left(x_{1}, x_{2}\right)$, and hence we have

$$
\xi\left(d\left(x_{2}, T x_{2}\right)\right) \leq \psi\left(\xi\left(d\left(x_{1}, x_{2}\right)\right)\right)<\psi^{2}(\xi(c)) .
$$

Hence, there exists $x_{3} \in T x_{2}$ such that

$$
\xi\left(d\left(x_{2}, x_{3}\right)\right)<\psi^{2}(\xi(c)) .
$$

Since $T$ is $\alpha$-admissible, from $x_{2} \in T x_{1}$ and $\alpha\left(x_{1}, x_{2}\right) \geq 1$, we have

$$
\alpha\left(x_{2}, x_{3}\right) \geq 1 .
$$

By induction, we obtain a sequence $\left\{x_{n}\right\} \subset X$ such that, for all $n \in \mathbb{N} \cup\{0\}$,

$$
\alpha\left(x_{n}, x_{n+1}\right) \geq 1,
$$

$x_{n+1} \in T x_{n}, x_{n} \neq x_{n+1}, \quad \xi\left(d\left(x_{n}, x_{n+1}\right)\right)<\psi^{n}(\xi(c))$.

Let $\epsilon>0$ be given.

Since $\sum_{n=0}^{\infty} \psi^{n}(\xi(c))<\infty$, there exists $N \in \mathbb{N}$ such that

$$
\sum_{n \geq N} \psi^{n}(\xi(c))<\xi(\epsilon) .
$$

For all $m>n \geq N$, we have

$$
\begin{aligned}
\xi\left(d\left(x_{n}, x_{m}\right)\right) & \leq \sum_{k=n}^{m-1} \psi^{k}(\xi(c)) \\
& <\sum_{n \geq N} \psi^{n}(\xi(c))<\xi(\epsilon)
\end{aligned}
$$

which implies $d\left(x_{n}, x_{m}\right)<\epsilon$ for all $m>n \geq N$. Hence, $\left\{x_{n}\right\}$ is a Cauchy sequence in $X$.

It follows from the completeness of $X$ that there exists

$$
x_{*}=\lim _{n \rightarrow \infty} x_{n} \in X .
$$

Suppose that $T$ is continuous.

We have

$$
\begin{aligned}
d\left(x_{*}, T x_{*}\right) & \leq d\left(x_{*}, x_{n+1}\right)+d\left(x_{n+1}, T x_{*}\right) \\
& \leq d\left(x_{*}, x_{n+1}\right)+H\left(x_{n}, T x_{*}\right) .
\end{aligned}
$$

By letting $n \rightarrow \infty$ in the above inequality, we obtain $d\left(x_{*}, T x_{*}\right)=0$, and so $x_{*} \in T x_{*}$.

Assume that $f_{T}$ is lower semicontinuous.

Then, $f_{T}\left(x_{*}\right) \leq \lim _{n \rightarrow \infty} f_{T}\left(x_{n}\right)$. Hence, $d\left(x_{*}, T x_{*}\right) \leq$ $\lim _{n \rightarrow \infty} d\left(x_{n}, T x_{n}\right) \leq \lim _{n \rightarrow \infty} d\left(x_{n}, x_{n+1}\right)=0$. Thus, $x_{*} \in$ $T x_{*}$.

Corollary 5. Let $(X, d)$ be a complete metric space, and let $\alpha$ : $X \times X \rightarrow[0, \infty)$ be a function. Suppose that $T: X \rightarrow C L(X)$ is an $\alpha$-admissible mapping.

Assume that, for all $x, y \in X$,

$$
\xi(\alpha(x, y) H(T x, T y)) \leq \psi(\xi(M(x, y)))+L \xi(d(y, T x)),
$$

where $L \geq 0, \xi \in \Xi$, and $\psi \in \Psi$ is strictly increasing.

Also, suppose that conditions (1) and (2) of Theorem 4 are satisfied.

Then $T$ has a fixed point in $X$. 
Remark 6. If we have $\xi(t)=t$ for all $t \geq 0, L=0$, and $T$ is continuous, then Corollary 5 reduces to Theorem 3.4 of [7].

Let $(X, \preceq)$ be an ordered set and $A, B \subset X$. We say that $A \preceq B$ whenever, for each $a \in A$, there exists $b \in B$ such that $a \preceq b$.

Corollary 7. Let $(X, \preceq, d)$ be a complete ordered metric space. Suppose that a multivalued mapping $T: X \rightarrow C L(X)$ satisfies

$$
\xi(H(T x, T y)) \leq \psi(\xi(M(x, y)))+L \xi(d(y, T x))
$$

for all $x, y \in X$ with $T x \preceq T y$ (resp., $T y \preceq T x$ ), where $L \geq 0$, $\xi \in \Xi$, and $\psi \in \Psi$ is strictly increasing.

Assume that, for each $x \in X$ and $y \in T x$ with $T x \preceq T y$ (resp., $T y \preceq T x$ ), we have $T y \preceq T z$ (resp., $T z \preceq T y$ ) for all $z \in T y$.

Also, suppose that the following are satisfied:

(1) there exists $x_{0} \in X$ and $x_{1} \in T x_{0}$ such that $T x_{0} \preceq T x_{1}$ (resp., $\left.T x_{1} \leq T x_{0}\right)$;

(2) either $T$ is continuous or $f_{T}$ is lower semicontinuous.

Then $T$ has a fixed point in $X$.

Remark 8. If we have $\xi(t)=t$ for all $t \geq 0, L=0$, and $T$ is continuous, then Corollary 7 reduces to Corollary 3.6 of [7].

From Theorem 4 we obtain the following result.

Corollary 9. Let $(X, d)$ be a complete metric space, and let $\alpha$ : $X \times X \rightarrow[0, \infty)$ be a function. Suppose that $T: X \rightarrow C L(X)$ is an $\alpha_{*}$-admissible mapping.

Assume that, for all $x, y \in X, \alpha(x, y) \geq 1$ implies

$$
\xi(H(T x, T y)) \leq \psi(\xi(M(x, y)))+L \xi(d(y, T x)),
$$

where $L \geq 0, \xi \in \Xi$, and $\psi \in \Psi$ is strictly increasing.

Also, suppose that conditions (1) and (2) of Theorem 4 are satisfied.

Then $T$ has a fixed point in $X$.

Remark 10. If we have $L=0$ in Corollary 9, then Corollary 9 reduces to Theorem 2.5 of [6].

Corollary 11. Let $(X, d)$ be a complete metric space, and let $\alpha$ : $X \times X \rightarrow[0, \infty)$ be a function. Suppose that $T: X \rightarrow C L(X)$ is an $\alpha_{*}$-admissible mapping.

Assume that, for all $x, y \in X$,

$$
\xi(\alpha(x, y) H(T x, T y)) \leq \psi(\xi(M(x, y)))+L \xi(d(y, T x)),
$$

where $L \geq 0, \xi \in \Xi$, and $\psi \in \Psi$ is strictly increasing.

Also, suppose that conditions (1) and (2) of Theorem 4 are satisfied.

Then $T$ has a fixed point in $X$.

Remark 12. In Corollary 11, let $\xi(t)=t$ for all $t \geq 0$ and $\alpha(x, y)=1$ for all $x, y \in X$ and $\psi(t)=k t$ for all $t \geq 0$, where $k \in[0,1)$. If $T$ is single valued map, then Corollary 11 reduces to Theorem 2.2 of [8].
Theorem 13. Let $(X, d)$ be a complete metric space, and let $\alpha$ : $X \times X \rightarrow[0, \infty)$ be a function. Suppose that a multivalued mapping $T: X \rightarrow C L(X)$ is $\alpha$-admissible.

Assume that, for all $x, y \in X, \alpha(x, y) \geq 1$ implies

$$
\xi(H(T x, T y)) \leq \psi(\xi(M(x, y)))+L \xi(d(y, T x)),
$$

where $L \geq 0, \xi \in \Xi$, and $\psi \in \Psi$ is strictly increasing and upper semicontinuous function. Also, suppose that the following are satisfied:

(1) there exists $x_{0} \in X$ and $x_{1} \in T x_{0}$ such that $\alpha\left(x_{0}, x_{1}\right) \geq$ 1 ;

(2) for a sequence $\left\{x_{n}\right\}$ in $X$ with $\alpha\left(x_{n}, x_{n+1}\right) \geq 1$ for all $n \in \mathbb{N} \cup\{0\}$ and a cluster point $x$ of $\left\{x_{n}\right\}$, there exists $a$ subsequence $\left\{x_{n(k)}\right\}$ of $\left\{x_{n}\right\}$ such that, for all $k \in \mathbb{N} \cup\{0\}$,

$$
\alpha\left(x_{n(k)}, x\right) \geq 1 \text {. }
$$

Then T has a fixed point in $X$.

Proof. Following the proof of Theorem 4, we obtain a sequence $\left\{x_{n}\right\} \subset X$ with $\lim _{n \rightarrow \infty} x_{n}=x_{*} \in X$ such that, for all $n \in \mathbb{N} \cup\{0\}$,

$$
x_{n+1} \in T x_{n}, \quad x_{n} \neq x_{n+1}, \quad \alpha\left(x_{n}, x_{n+1}\right) \geq 1 .
$$

From (2) there exists a subsequence $\left\{x_{n(k)}\right\}$ of $\left\{x_{n}\right\}$ such that

$$
\alpha\left(x_{n(k)}, x_{*}\right) \geq 1 \text {. }
$$

Thus, we have

$$
\begin{aligned}
\xi\left(d\left(x_{n(k)+1}, T x_{*}\right)\right)= & \xi\left(H\left(T x_{n(k)}, T x_{*}\right)\right) \\
\leq & \psi\left(\xi\left(M\left(x_{n(k)}, x_{*}\right)\right)\right) \\
& +L \xi\left(d\left(x_{*}, x_{n(k)+1}\right)\right),
\end{aligned}
$$

where

$$
\begin{aligned}
M\left(x_{n(k)}, x_{*}\right) & \\
=\max & \left\{d\left(x_{n(k)}, x_{*}\right), d\left(x_{n(k)}, x_{n(k)+1}\right), d\left(x_{*}, T x_{*}\right),\right. \\
& \left.\frac{1}{2}\left\{d\left(x_{n(k)}, T x_{*}\right)+d\left(x_{*}, x_{n(k)+1}\right)\right\}\right\} .
\end{aligned}
$$

We have

$$
\lim _{k \rightarrow \infty} M\left(x_{n(k)}, x_{*}\right)=d\left(x_{*}, T x_{*}\right),
$$

and so

$$
\lim _{k \rightarrow \infty} \xi\left(M\left(x_{n(k)}, x_{*}\right)\right)=\xi\left(d\left(x_{*}, T x_{*}\right)\right) .
$$

Suppose that $d\left(x_{*}, T x_{*}\right) \neq 0$.

Since $\psi$ is upper semicontinuous,

$$
\lim _{k \rightarrow \infty} \psi\left(\xi\left(M\left(x_{n(k)}, x_{*}\right)\right)\right) \leq \psi\left(\xi\left(d\left(x_{*}, T x_{*}\right)\right)\right) .
$$


Letting $k \rightarrow \infty$ in inequality (29) and using continuity of $\xi$, we obtain

$$
\begin{aligned}
0 & <\xi\left(d\left(x_{*}, T x_{*}\right)\right) \\
& \leq \lim _{k \rightarrow \infty} \psi\left(\xi\left(M\left(x_{n(k)}, x_{*}\right)\right)\right)+\lim _{k \rightarrow \infty} L \xi\left(d\left(x_{*}, x_{n(k)+1}\right)\right) \\
& \leq \psi\left(\xi\left(d\left(x_{*}, T x_{*}\right)\right)\right) \\
& <\xi\left(d\left(x_{*}, T x_{*}\right)\right)
\end{aligned}
$$

which is a contradiction. Hence, $d\left(x_{*}, T x_{*}\right)=0$, and hence $x_{*}$ is a fixed point of $T$.

The following example shows that upper semicontinuity of $\psi$ cannot be dropped in Theorem 13 .

Example 14. Let $X=[0, \infty)$, and let $d(x, y)=|x-y|$ for all $x, y \geq 0$.

Define a mapping $T: X \rightarrow C L(X)$ by

$$
T x= \begin{cases}\left\{\frac{1}{2}, 1\right\} & (x=0), \\ \left\{\frac{3}{4} x\right\} & (0<x \leq 1), \\ \{2 x\} & (x>1) .\end{cases}
$$

Let $\xi(t)=t$ for all $t \geq 0$, and let

$$
\psi(t)= \begin{cases}\frac{4}{5} t & (t \geq 1), \\ \frac{3}{4} t & (0 \leq t<1) .\end{cases}
$$

Then, $\xi \in \Xi$, and $\psi \in \Psi$ and $\psi$ is a strictly increasing function.

Let $\alpha, \eta: X \times X \rightarrow[0, \infty)$ be defined by

$$
\alpha(x, y)= \begin{cases}4 & (0 \leq x, y \leq 1) \\ 0 & \text { otherwise }\end{cases}
$$

Obviously, condition (2) of Theorem 13 is satisfied. Condition (1) of Theorem 13 is satisfied with $x_{0}=1 / 4$.

We show that (7) is satisfied.

Let $x, y \in X$ be such that $\alpha(x, y) \geq 1$.

Then, $0 \leq x, y \leq 1$.

If $x=y$, then obviously (7) is satisfied.

Let $x \neq y$.

If $x=0$ and $0<y \leq 1$, then we obtain

$$
\begin{aligned}
\xi(H(T x, T y)) & =H\left(\left\{\frac{1}{2}, 1\right\}, \frac{3}{4} y\right) \\
& \leq \frac{1}{4} \leq \psi(d(x, T x)) \leq \psi(\xi(M(x, y))) .
\end{aligned}
$$

Let $0<x \leq 1$ and $0<y \leq 1$.
Then, we have

$$
\begin{aligned}
\xi(H(T x, T y)) & =d(T x, T y)=d\left(\frac{3}{4} x, \frac{3}{4} y\right) \\
& =\frac{3}{4}|x-y|=\psi(d(x, y)) \\
& \leq \psi(\xi(M(x, y))) .
\end{aligned}
$$

Thus, (7) is satisfied.

We now show that $T$ is $\alpha$-admissible.

Let $x \in X$ be given, and let $y \in T x$ be such that $\alpha(x$, $y) \geq 1$.

Then, $0 \leq x, y \leq 1$.

Obviously, $\alpha(y, z) \geq 1$ for all $z \in T y$ whenever $0<y \leq 1$.

If $y=0$, then $T y=\{1 / 2,1\}$. Hence, for all $z \in T y$, $\alpha(y, z) \geq 1$.

Hence, $T$ is $\alpha$-admissible. Thus, all hypotheses of Theorem 13 are satisfied. However, $T$ has no fixed points.

Note that $\psi$ is not upper semicontinuous.

Corollary 15. Let $(X, d)$ be a complete metric space, and let $\alpha$ : $X \times X \rightarrow[0, \infty)$ be a function. Suppose that $T: X \rightarrow C L(X)$ is an $\alpha$-admissible mapping.

Assume that, for all $x, y \in X$,

$$
\xi(\alpha(x, y) H(T x, T y)) \leq \psi(\xi(M(x, y)))+L \xi(d(y, T x)),
$$

where $L \geq 0, \xi \in \Xi$, and $\psi \in \Psi$ is strictly increasing and upper semicontinuous function. Also, suppose that conditions (1) and (2) of Theorem 13 are satisfied.

Then $T$ has a fixed point in $X$.

Corollary 16. Let $(X, \preceq, d)$ be a complete ordered metric space. Suppose that a multivalued mapping $T: X \rightarrow C L(X)$ satisfies

$$
\xi(H(T x, T y)) \leq \psi(\xi(M(x, y)))+L \xi(d(y, T x))
$$

for all $x, y \in X$ with $T x \preceq T y$ (resp., Ty $\preceq T x$ ), where $L \geq 0, \xi \in \Xi$, and $\psi \in \Psi$ is strictly increasing and upper semicontinuous function.

Assume that, for each $x \in X$ and $y \in T x$ with Tx $\preceq$ Ty (resp., $T y \preceq T x$ ), we have $T y \preceq T z$ (resp., $T z \preceq T y$ ) for all $z \in T y$.

Also, suppose that the following are satisfied:

(1) there exists $x_{0} \in X$ and $x_{1} \in T x_{0}$ such that $T x_{0} \preceq T x_{1}$ (resp., $\left.T x_{1} \preceq T x_{0}\right)$;

(2) for a sequence $\left\{x_{n}\right\}$ in $X$ with $x_{n} \preceq x_{n+1}$ (resp., $x_{n+1} \preceq$ $x_{n}$ ) for all $n \in \mathbb{N} \cup\{0\}$ and a cluster point $x$ of $\left\{x_{n}\right\}$, there exists a subsequence $\left\{x_{n(k)}\right\}$ of $\left\{x_{n}\right\}$ such that, for all $k \in \mathbb{N} \cup\{0\}$,

$$
x_{n(k)} \preceq x \quad\left(\text { resp., } x \preceq x_{n(k)}\right) .
$$

Then $T$ has a fixed point in X.

Remark 17. Corollary 16 is a generalization and extension of the result of [9] to multivalued mappings. 
Corollary 18. Let $(X, d)$ be a complete metric space, and let $\alpha: X \times X \rightarrow[0, \infty)$ be a function. Suppose that a multivalued mapping $T: X \rightarrow C L(X)$ is $\alpha_{*}$-admissible.

Assume that, for all $x, y \in X, \alpha(x, y) \geq 1$ implies

$$
\xi(H(T x, T y)) \leq \psi(\xi(M(x, y)))+L \xi(d(y, T x)),
$$

where $L \geq 0, \xi \in \Xi$, and $\psi \in \Psi$ is strictly increasing and upper semicontinuous function.

Also, suppose that conditions (1) and (2) of Theorem 13 are satisfied.

Then $T$ has a fixed point in $X$.

Remark 19. By taking $L=0$ in Corollary 18 and by applying Remark 1, Corollary 18 reduces to Theorem 2.6 of [6].

Corollary 20. Let $(X, d)$ be a complete metric space, and let $\alpha: X \times X \rightarrow[0, \infty)$ be a function. Suppose that $T: X \rightarrow$ $C L(X)$ is an $\alpha_{*}$-admissible mapping.

Assume that, for all $x, y \in X$,

$$
\xi(\alpha(x, y) H(T x, T y)) \leq \psi(\xi(M(x, y)))+L \xi(d(y, T x)),
$$

where $L \geq 0, \xi \in \Xi$, and $\psi \in \Psi$ is strictly increasing and upper semicontinuous function.

Also, suppose that conditions (1) and (2) of Theorem 13 are satisfied.

Then $T$ has a fixed point in $X$.

\section{Conflict of Interests}

The author declares that there is no conflict of interests regarding the publication of this paper.

\section{Acknowledgment}

The author would like to thank the anonymous reviewers for their valuable comments.

\section{References}

[1] B. Samet, C. Vetro, and P. Vetro, "Fixed point theorems for $\alpha-\psi$-contractive type mappings," Nonlinear Analysis: Theory, Methods \& Applications, vol. 75, no. 4, pp. 2154-2165, 2012.

[2] J. H. Asl, S. Rezapour, and N. Shahzad, "On fixed points of $\alpha-\psi$-contractive multifunctions," Fixed Point Theory and Applications, vol. 2012, article 212, 2012.

[3] P. Salimi, A. Latif, and N. Hussain, "Modified $\alpha-\psi$-contractive mappings with applications," Fixed Point Theory and Applications, vol. 2013, article 151, 2013.

[4] E. Karapinar and B. Samet, "Generalized $\alpha-\psi$ contractive type mappings and related fixed point theorems with applications," Abstract and Applied Analysis, vol. 2012, Article ID 793486, 17 pages, 2012.

[5] N. Hussain, P. Salimi, and A. Latif, "Fixed point results for single and set-valued $\alpha-\eta-\psi$-contractive mappings," Fixed Point Theory and Applications, vol. 2013, article 212, 2013.

[6] M. U. Ali, T. Kamran, and E. Karapınar, " $(\alpha, \psi, \xi)$-contractive multivalued mappings," Fixed Point Theory and Applications, vol. 2014, article 7, 2014.
[7] B. Mohammadi, S. Rezapour, and N. Shahzad, "Some results on fixed points of $\alpha-\psi$-Ciric generalized multifunctions," Fixed Point Theory and Applications, vol. 2013, article 24, 2013.

[8] V. Berinde, "Some remarks on a fixed point theorem for Cirictype almost contractions," Carpathian Journal of Mathematics, vol. 25, no. 2, pp. 157-162, 2009.

[9] R. P. Agarwal, M. A. El-Gebeily, and D. O'Regan, "Generalized contractions in partially ordered metric spaces," Applicable Analysis, vol. 87, no. 1, pp. 109-116, 2008. 


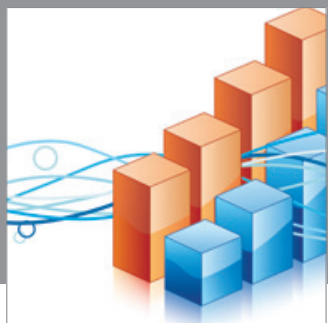

Advances in

Operations Research

mansans

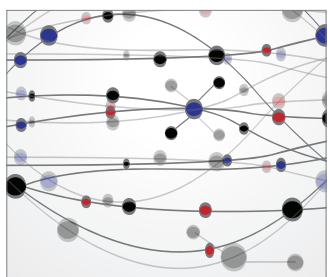

The Scientific World Journal
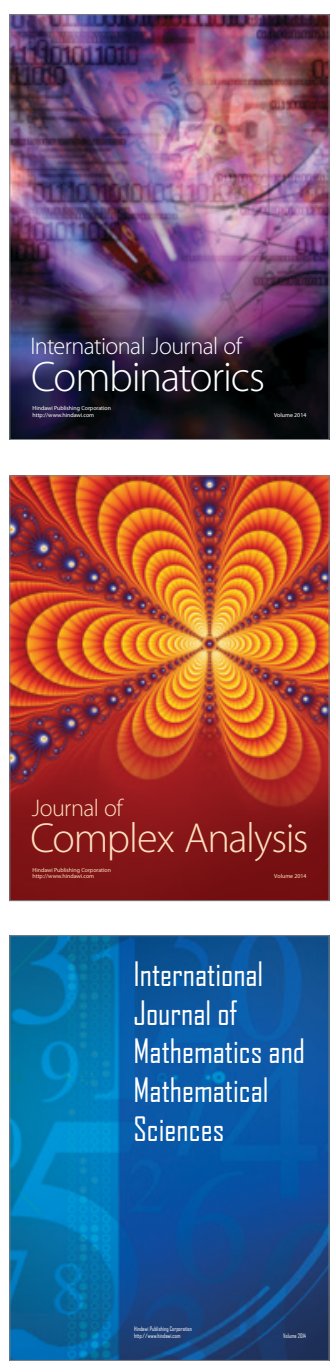
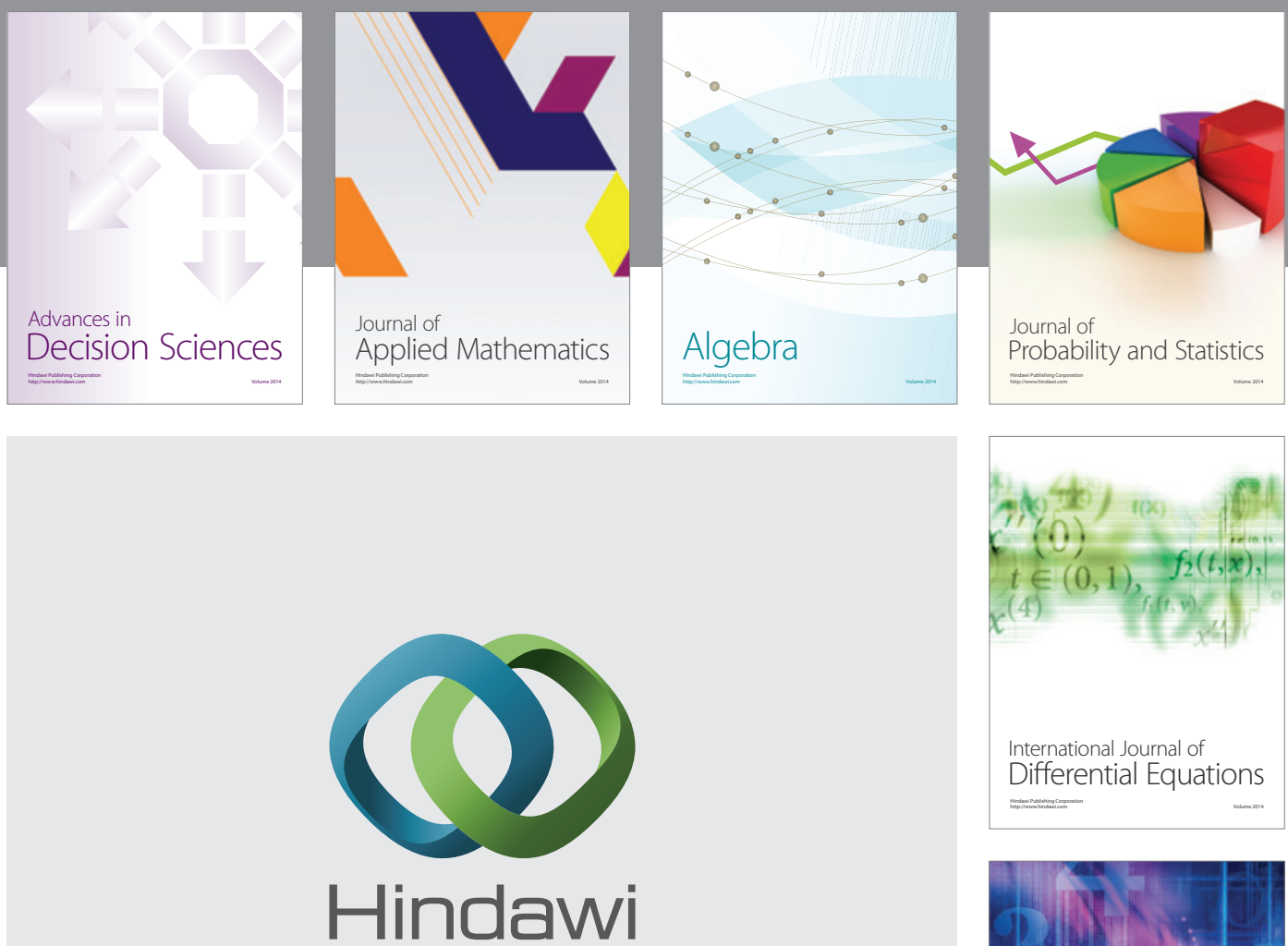

Submit your manuscripts at http://www.hindawi.com
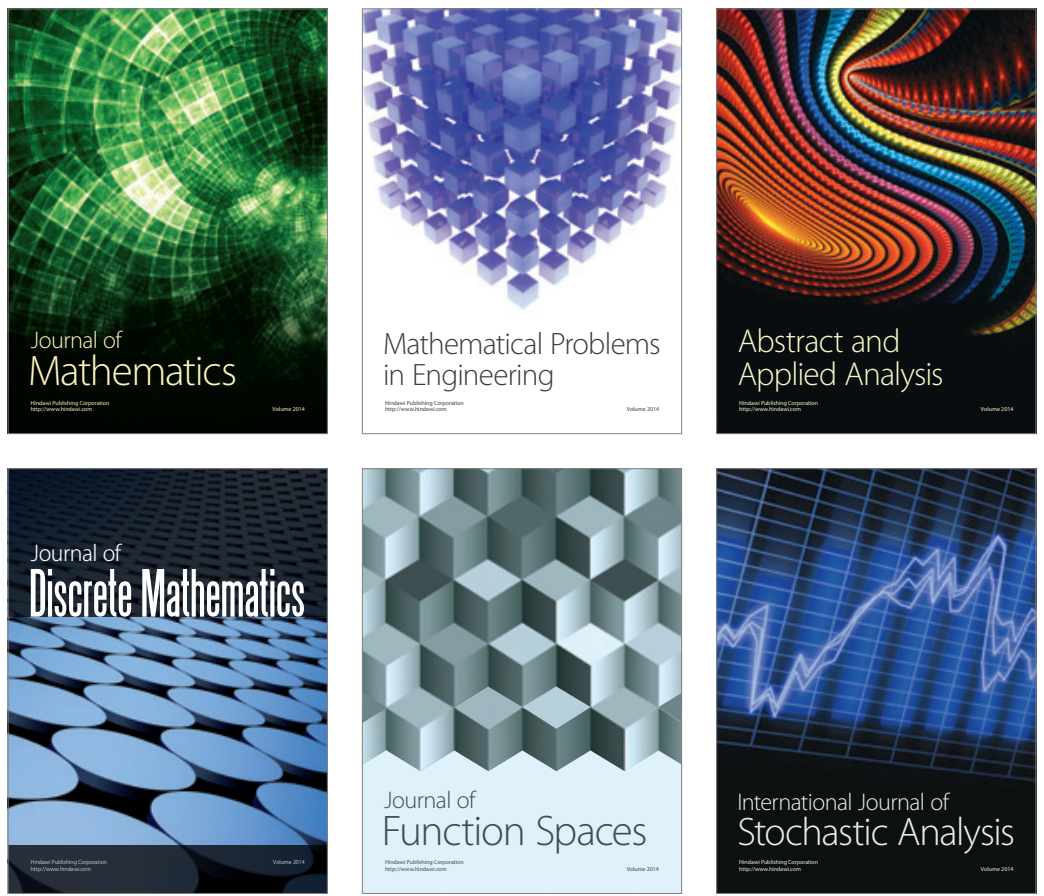

Journal of

Function Spaces

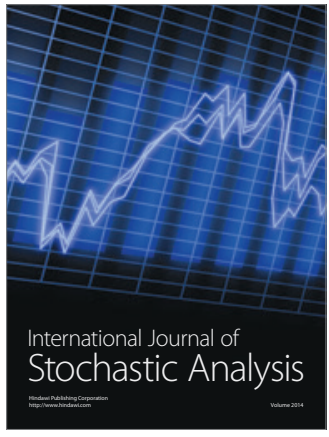

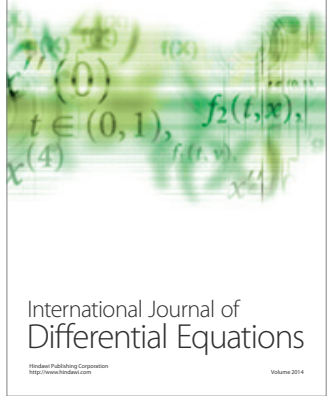
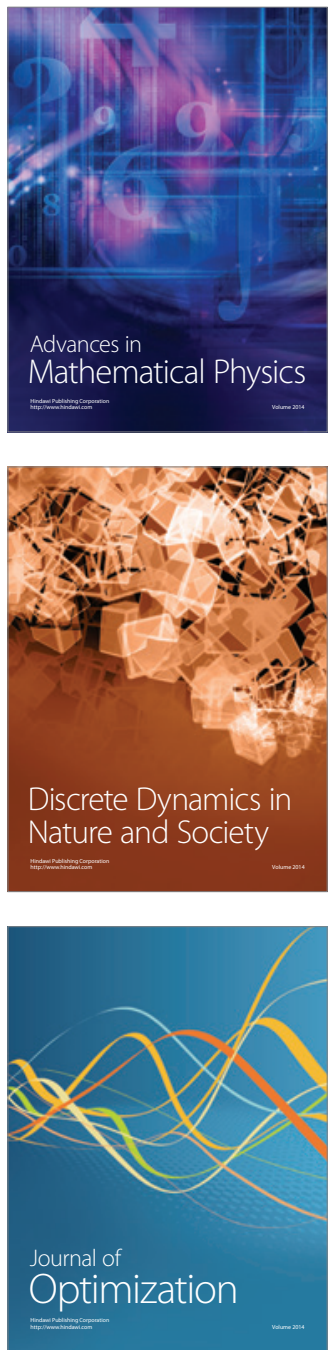